\title{
High-Quality White Light Generation using Dually Hybridized Nanocrystals and Conjugated Polymers
}

\author{
Sedat Nizamoglu ${ }^{1,2}$, Tuncay Ozel ${ }^{1,2}$, Evren Mutlugun ${ }^{1,2}$, Ilkem Ozge Huyal, ${ }^{1,4}$ Emre Sari ${ }^{1,3}$, Nan Tian ${ }^{5}$, \\ Elisabeth Holder ${ }^{5}$, and Hilmi Volkan Demir ${ }^{1,2,3}$ \\ ${ }^{I}$ Devices and Sensors Group, Nanotechnology Research Center; ${ }^{2}$ Department of Physics; \\ ${ }^{3}$ Department of Electrical and Electronics Engineering; and ${ }^{4}$ Department of Chemistry, Bilkent University, Ankara 06800 Turkey \\ ${ }^{5}$ Functional Polymers Group and Institute of Polymer Technology, University of Wuppertal, Gaußstrasse 20, D-42097 Wuppertal, Germany
}

For solid state lighting, III-nitride based light emitting diodes (LEDs) coated with phosphors are commonly used [1]. In such integrated white LEDs (WLEDs), the white light is generated by means of electroluminescence (EL) from the LED (e.g., in blue) and photoluminescence (PL) from the luminescent phosphors (e.g., in yellow) on the top (serving for color conversion) pumped by the integrating LED platform. Although phosphors are good color-converting material because of their broad-band photoemission, difficulties in controlling their granule size and in mixing and depositing their uniform films typically cause undesired visible color variations, posing important problems for wide-scale applications [2]. Additionally, it is not easy to increase the color rendering index for the purpose of high-quality lighting by using such phosphor based color conversion. In general, yellow phosphor based WLEDs exhibit color rendering indices of about 70, but a color rendering index above 80 is required for the future solid-state lighting systems as road-mapped by Sandia National Laboratories [3]. Also, it is not a simple task to reshape phosphors emission spectrum and to tune the properties of the resulting white light such as the tristimulus coordinates and color temperature. However, such tuning may be necessary to optimize the generated light for the lighting conditions of different applications (for example, for museum displays, shop windows, vehicles, etc.). Organic-inorganic hybridized LEDs that incorporate fluorescent conjugated polymer and nanocrystal (NC) emitters for color conversion hold great promise to overcome these difficulties and obtain the desired high-quality white light. To this end, in this work, we developed and demonstrated layer-by-layer assembly of highly luminescent $\mathrm{CdSe} / \mathrm{ZnS}$ nanocrystals closely packed in multiple combinations on highly fluorescent polyfluorene that are dually hybridized on near-UV emitting nitride based LEDs to generate highquality white light with color rendering index $>80$ and to tune the white light properties as desired (Figure 1).

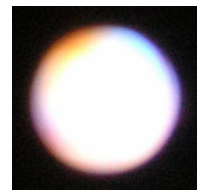

(a)

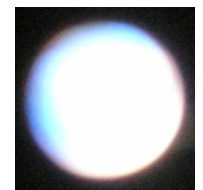

(b)

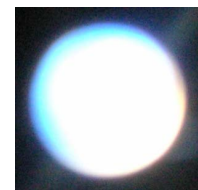

(c)

Figure 1. Images of white light emission from electrically driven nanocrystal-conjugated polymer hybridized WLEDs incorporating: (a) yellow NCs on blue polyfluorene, (b) yellow-green NCs on blue polyfluorene, and (c) yellow-green-red NCs on blue polyfluorene, all hybridized on n-UV LEDs.

We produced blue emitting 9,9-bis (2-ethylhexyl) polyfluorene as the fluorescent conjugated polymer by following a synthetic protocol according to Yamamoto [4, 5-7]. The photoluminescence peak of the polyfluorene was at $439 \mathrm{~nm}$, with a PL quantum yield of about $45 \%$. Its molecular weigth was $\mathrm{Mn}=42000 \mathrm{~g} / \mathrm{mol}, \mathrm{Mw}=$ $79000 \mathrm{~g} / \mathrm{mol}$, giving a polydispersity index of 1.88 . We also prepared high-concentration CdSe/ZnS NC solutions and evaporated NC films for optimal film formation. The emission colors of these NCs were green $(540 \mathrm{~nm})$, yellow $(580 \mathrm{~nm})$, and red $(620 \mathrm{~nm})$ tuned using the quantum size effect. Their crystal diameters were $2.4 \mathrm{~nm}, 3.2 \mathrm{~nm}$, and $5.2 \mathrm{~nm}$ (with a size distribution of $\pm 5 \%$ ) with the corresponding molecular weights of 14 $\mu \mathrm{g} / \mathrm{nmol}, 38 \mu \mathrm{g} / \mathrm{nmol}$, and $180 \mu \mathrm{g} / \mathrm{nmol}$. We implemented all hybrid devices on near UV (n-UV) InGaN/GaN based LED pump sources (emitting at $383 \mathrm{~nm}$ ) grown on a $c$-plane sapphire using our metal organic chemical vapor deposition (MOCVD) system. We followed standard microfabrication processing that included photolithography, thermal evaporation (metallization), reactive ion etch (RIE), and rapid thermal annealing described in our other work [8-10].

In this work, to tune the white light properties, we combined layer-by-layer assembly of closely packed $\mathrm{CdSe} / \mathrm{ZnS}$ core-shell nanocrystals and polyfluorene conjugated polymer hybridized on n-UV emitting nitride based LEDs. First, we experimentally realized layer-by-layer hybridization of yellow nanocrystals $\left(\lambda_{\mathrm{PL}}=580 \mathrm{~nm}\right)$ and polyfluorene $\left(\lambda_{\mathrm{PL}}=439 \mathrm{~nm}\right)$ to obtain tristimulus coordinates of $(x, y)=(0.31,0.27)$, correlated color temperature of $T_{\mathrm{c}}=6962 \mathrm{~K}$ and color rendering index of $R_{\mathrm{a}}=53.4$ as shown in Figure 1 (a). Second, we implemented layer-by-layer assembly of yellow and green nanocrystals $\left(\lambda_{\mathrm{PL}}=580\right.$ and $\left.540 \mathrm{~nm}\right)$ and blue polyfluorene $\left(\lambda_{\mathrm{PL}}=439 \mathrm{~nm}\right)$ with $(x, y)=(0.23,0.30), T_{\mathrm{c}}=14395 \mathrm{~K}$ and $R_{\mathrm{a}}=65.7$ as shown in Figure 1 (b); and finally we attained layer-by-layer deposition of yellow, green, and red nanocrystals $\left(\lambda_{\mathrm{PL}}=580,540\right.$ and $\left.620 \mathrm{~nm}\right)$ and blue polyfluorene $\left(\lambda_{\mathrm{PL}}=439 \mathrm{~nm}\right)$ with $(x, y)=(0.38,0.39), T_{\mathrm{c}}=4052 \mathrm{~K}$ and $R_{\mathrm{a}}=83.0$ as shown in Figure 1 (c). In addition to the nanocyrstals emitting in red, yellow and green, the polyfluorene provided efficient emission in blue, which is particularly beneficial due to the difficulty to obtain high emission efficiency at shorter 
wavelengths using the NCs (e.g., in blue). By utilizing both polyfluorene and nanocrystals, it was possible to control and improve the color rendering index to values higher than 80 as desired, with the increasing multichromaticity of the nanocrystal and polymer emitters.

To get an enhanced control of the color rendering index, we also monitored the color properties of the resulting hybrid LEDs step by step after each emitter layer was hybridized. The final hybrid device that incorporated yellow-green-red NCs on blue polyfluorene was carefully designed and realized in steps to exhibit high light quality. First, a $1.03 \mu \mathrm{m}$ thick layer of $3 \mathrm{mg} / \mathrm{ml}$ blue polymer was hybridized on top of the InGaN/GaN n-UV $\operatorname{LED}\left(\lambda_{\mathrm{EL}}=383 \mathrm{~nm}\right)$ (called Sample 1 shown in Figure 2 (a)). At this point, the electroluminescence of the LED along with fluorescence of the blue polymer resulted in tristimulus coordinates of $\mathrm{x}=0.18$ and $\mathrm{y}=0.21$, a correlated color temperature of $T_{c}=34463 \mathrm{~K}$, and a color rendering index of $R_{a}=49.5$. Subsequently, on the polymer surface, closely-packed green NCs ( $\sim 400 \mathrm{~nm}$ thick) were integrated. The optical properties were then observed to shift to $\mathrm{x}=0.32$ and $\mathrm{y}=0.37$ falling into the white region, with $\mathrm{R}_{\mathrm{a}}$ increasing to 66.0 and $\mathrm{T}_{\mathrm{c}}$ decreasing to $5896 \mathrm{~K}$ (Sample 2). With the addition of these green NCs, the color rendering index was enhanced by $\sim 16$ units and the color temperature was reduced by $28567 \mathrm{~K}$ moving towards the planckian sources on the red side. Later when the device was further hybridized with closely-packed yellow NCs $(\sim 135 \mathrm{~nm}$ thick), the optical properties were obtained as $\mathrm{x}=0.32, \mathrm{y}=0.37, \mathrm{~T}_{\mathrm{c}}=5694 \mathrm{~K}$, and $\mathrm{R}_{\mathrm{a}}=73.7$ (Sample 3). As a result, the color rendering index reached to 73.7 and the correlated color temperature further decreased to $5694 \mathrm{~K}$. Finally, closely-packed red NCs ( $292 \mathrm{~nm}$ thick) were incorporated on the very top, leading to an improved color rendering index of $R_{\mathrm{a}}=83.0$ and a decreased correlated color temperature of $T_{\mathrm{c}}=4052 \mathrm{~K}$ at the operating point of $\mathrm{x}=0.38, \mathrm{y}=0.39$ (Sample 4). Starting from Sample 1 and ending with Sample 4, Figure 2 (a) shows the progress of the color rendering index tuning. Here a well-controlled color rendering index tuning is possible with an improvement from 49.5 to 83.0 by the controlled layer-by-layer assembly of NCs and polyfluorene. Figure 2 (b) displays the electroluminescence spectra of Sample 4 when different levels of current are injected to the WLED at room temperature. Here an image of the generated white light and the location of the $(x, y)$ coordinates are also presented. Consequently, the future lighting requirement was fulfilled by demonstrating a color rendering index higher than 80 using dual hybridization of nanocrystal and polymer emitters, which is not the case for yellow phosphor coated blue LEDs typically with a low color rendering index of about 70. [10]
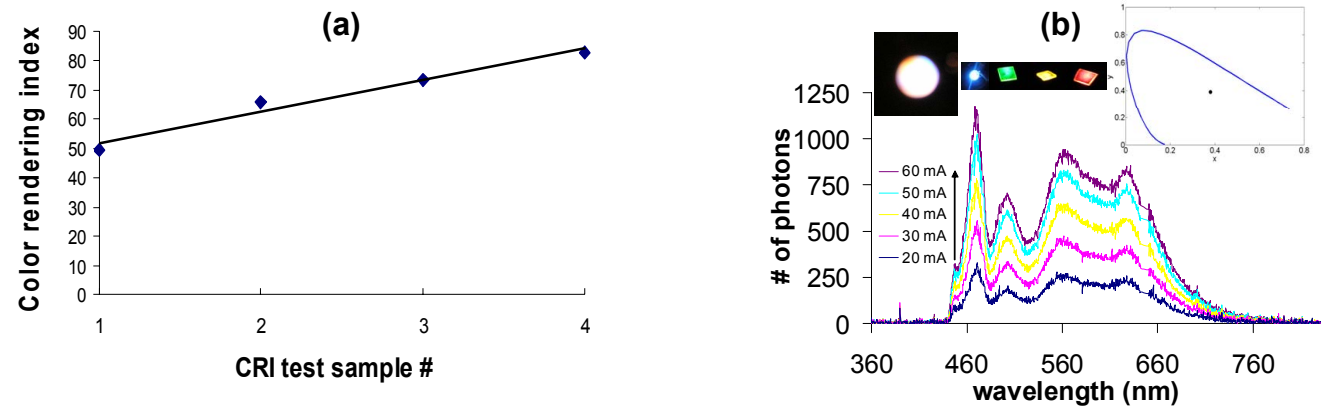

Figure 2. (a) Tuning color rendering index from Sample 1 to 4 by controlling the layer-by-layer assembly of polyfluorene and NC emitters and (b) emission spectra of blue polyfluorene and yellow-green-red CdSe/ZnS core-shell nanocrystals layer-by-layer hybridized on an n-UV InGaN/GaN LED at different levels of current injection at room temperature along with the pictures of generated lights and location of the corresponding operating $(x, y)$ coordinates.

In conclusion, hybridizing different sizes of $\mathrm{CdSe} / \mathrm{ZnS}$ core-shell nanocrystal emitters layer by layer with highly fluorescent polyfluorene as conjugated polymer on $n-U V$ InGaN/GaN LEDs, we achieved high-quality white light with color rendering index $>80$, fulfilling the future solid state lighting requirement. Here we also demonstrated the ability to tune the white light properties as desired by carefully designing and realizing the hybrid device parameters including the type and film thickness of closely-packed NC emitters, the concentration and film thickness of polymer emitters, and the order of NC and polymer films. Given their high light quality and tunable optical properties, these hybrid organic-inorganic white light sources provide a high potential for future lighting and display applications.

Acknowledgements: This work is supported by EU FP6 PHOREMOST Network of Excellence 511616, EU FP6 Marie Curie European Reintegration Grant MOON 021391, TUBITAK EEEAG No. 106E020, 104E114, 105E065, and 105E066, TUBA GEBIP, and TUBITAK Fellowship programs. Prof. Dr. Ullrich Scherf and Prof. Dr. Ekmel Özbay are also kindly acknowledged for their supports.

References:

[1] E. F. Schubert, Cambridge University Press, 2006.

[2] G. Heliotis et al., J. Opt. A: Pure Appl. Opt., 8, 445, 2006.

[3] J. Y. Tsao et al., IEEE Circuits and Devices, 20, 3, 2004

[4] S. Tasch et al., Appl. Phys. Lett., 71, 2883, 1997.

[5] E. J. W. List et al., Opt. Mater,. 9, 183, 1998.

[6] E. J. W. List Leising et al., Jap. J. Appl. Phys. Part 2: Lett.,

[7] D. Neher et al., Macromol. Rapid Commun., 22, 1365, 2001. [8] S. Nizamoglu et al., Nanotechnology, 18, 065709, 2007.

[9] S. Nizamoglu et al., J. Opt. A: Pure Appl. Opt, 2007 (in press).

[10] H. V. Demir et al., New J. of Phys., 2007 (submitted). 